\title{
A LOCAL THERMODYNAMIC CRITERION FOR THE REFERENCE-HYPERNETTED CHAIN EQUATION
}

\author{
F. LADO \\ Department of Physics, North Carolina State University, Raleigh, NC 27650, USA
}

Received 18 January 1982

Rosenfeld and Ashcroft have demonstrated that the function $B_{0}(r)$, contained in the closure of the RHNC equation, can be adapted from the hard sphere model to produce excellent results for any potential. We propose here a local thermodynamic criterion for the best $B_{0}(r)$ based on minimizing the free energy.

The reference-hypernetted chain (RHNC) approximation [1] uses the closure

$C(r)=h(r)-\ln g(r) \mathrm{e}^{\beta \phi(r)}+B_{0}(r)$

with the Ornstein-Zernike equation defining the direct correlation function $C(r)$ in terms of the pair distribution function $g(r)$,

$\tilde{C}(k)=\tilde{h}(k) /[1-\rho \tilde{h}(k)]$,

where $h(r) \equiv g(r)-1$ and $\tilde{h}(k)$ is its Fourier transform. In eq. (1), $B_{0}(r)$ is the bridge function of a reference system with potential $\phi_{0}(r)$, usually just the short range, repulsive part of the full potential $\phi(r)$.

Rosenfeld and Ashcroft [2] have given a powerful reinterpretation of eq. (1). Arguing that the bridge functions $B(r)$ should have roughly the same functional form for all potentials, they proposed looking upon $B_{0}(r)$ in (1) as an adjustable function, choosing it from the hard sphere variety for some optimum sphere diameter $\sigma$. For the criterion of this choice, they suggested requiring consistency between the equations of state obtained separately from the virial theorem and the compressibility. This is indeed a strong condition that has led to improved results with other approximations [3] and also works well [4] with eq. (1). It is, though, an awkward constraint in that solutions must be obtained systematically along an entire isotherm, even if one is interested in just a single thermodynamic state. Furthermore, it cannot be applied at all if the isotherm passes through a two- phase region, i.e., precisely for true liquids. In this note, a strictly local thermodynamic condition is proposed that is free of these drawbacks.

The difference between the free energy of the fluid with intermolecular potential $\phi(r)$ and that of a reference fluid with potential $\phi_{0}(r)$ may be written [1]

$\beta \Delta A / N=\beta \Delta A_{1} / N+\beta \Delta A_{2} / N+\beta \Delta A_{3} / N$,

where

$\beta \Delta A_{1} / N=\beta A_{1} / N-\beta A_{1}^{(0)} / N$,
$\beta \Delta A_{2} / N=\beta A_{2} / N-\beta A_{2}^{(0)} / N$,
$\beta \Delta A_{3} / N=-\frac{1}{2} \rho \int \mathrm{d} r \int_{0}^{1} \mathrm{~d} \xi B(r ; \xi) \partial g(r ; \xi) / \partial \xi$,

with

$$
\begin{aligned}
\beta A_{1} / N & =-\frac{1}{2} \rho \int \mathrm{d} r\left[\frac{1}{2} h^{2}(r)+h(r)\right. \\
\left.-g(r) \ln g(r) \mathrm{e}^{\beta \phi(r)}\right] & \\
\beta A_{2} / N & =-\frac{1}{2 \rho} \int \frac{\mathrm{d} k}{(2 \pi)^{3}}\{\ln [1+\rho \tilde{h}(k)]-\rho \tilde{h}(k)\},
\end{aligned}
$$

and similar expressions for the reference fluid quantities $A_{1}^{(0)}$ and $A_{2}^{(0)}$. The RHNC approximation is based on the assumption that the bridge function $B(r ; \xi)$ in eq. (6) does not change significantly as the potential is varied from the reference $\phi_{0}(r)$ at $\xi=0$ to the full $\phi(r)$ at $\xi=1$; in this case, eq. (6) becomes 


$$
\beta \Delta A_{3} / N \approx-\frac{1}{2} \rho \int \mathrm{d} r B_{0}(r)\left[g(r)-g_{0}(r)\right],
$$

and a final, computable expression is obtained for the free energy. As shown in ref. [1], requiring that this expression be extremal with respect to variations in $g(r)$ yields the RHNC closure, eq. (1). We now adopt Rosenfeld and Ashcroft's view of the reference system as adjustable and require additionally that the free energy be extremal with respect to variations in $B_{0}(r)$ as well.

From equations (3) $-(6)$, we have

$\beta A / N=\beta A_{1} / N+\beta A_{2} / N+\beta A_{3}^{(0)} / N+\beta \Delta A_{3} / N$,

where the reference system quantity $A_{3}^{(0)}$ may be written

$\beta A\}^{(0)} / N=-\frac{1}{2} \rho \int \mathrm{d} r \int_{0}^{1} \mathrm{~d} \xi B_{0}(r ; \xi) \partial g_{0}(r ; \xi) / \partial \xi$,

with the "charging" parameter $\xi$ here applied to the entire potential $\phi_{0}(r)$. The contributions $A_{1}$ and $A_{2}$ are defined in (7) and (8) and for $\Delta A_{3}$ we will use the approximate eq. (9). Then variation of all the functions in these expressions $\left(g, g_{0}\right.$, and $\left.B_{0}\right)$ yields finally

$$
\begin{aligned}
& \beta \delta A / N=\frac{1}{2} \rho \int \mathrm{d} r\left[C(r)-h(r)+\ln g(r) \mathrm{e}^{\beta \phi(r)}\right. \\
& \left.\quad-B_{0}(r)\right] \delta g(r)-\frac{1}{2} \rho \int \mathrm{d} r\left[g(r)-g_{0}(r)\right] \delta B_{0}(r) .
\end{aligned}
$$

The first integral vanishes because of (1). For minimal free energy we thus require

$\rho \int \mathrm{d} r\left[g(r)-g_{0}(r)\right] \delta B_{0}(r)=0$.

In practice, completely general variations of the reference system cannot be managed. Instead, one would settle on a particular potential function

$\phi_{0}(r)=\phi_{0}(r ; \epsilon, \sigma)$

containing typically two parameters, $\epsilon$ and $\sigma$, which set the energy and distance scales. With the function $\phi_{0}$ chosen, it is these parameters which may be varied, so that condition (13) becomes

$I_{1} \equiv \rho \int \mathrm{d} r\left[g(r)-g_{0}(r)\right] \epsilon \partial B_{0}(r ; \epsilon, \sigma) / \partial \epsilon=0$,
$I_{2} \equiv \rho \int \mathrm{d} r\left[g(r)-g_{0}(r)\right] \sigma \partial B_{0}(r ; \epsilon, \sigma) / \partial \sigma=0$.

These then are the constraints for selection of the reference function $B_{0}(r ; \epsilon, \sigma)$; the optimum values of $\epsilon$ and $\sigma$ occur when these integrals vanish, leading to a minimum of the excess free energy functional (10). If the hard core potential is chosen as the reference system, eq. (15a) is of course automatically satisfied and only one variation remains.

It was noted in ref. [1] that an inconsistency appeared when the thermodynamics of the system was obtained from the approximate free energy (10). Explicitly, differentiation of $(10)$ gives for the internal energy $U$ and pressure $p$

$$
\begin{aligned}
& \beta U / N=\beta \partial(\beta A / N) / \partial \beta=\frac{1}{2} \rho \int \mathrm{d} r g(r) \beta \phi(r) \\
& -\frac{1}{2} \rho \int \mathrm{d} r\left[g(r)-g_{0}(r)\right] \beta \partial B_{0}(r) / \partial \beta, \\
& \beta p / \rho-1=\rho \partial(\beta A / N) / \partial \rho=-\frac{1}{6} \rho \int \mathrm{d} r g(r) r \beta \phi^{\prime}(r) \\
& +\frac{1}{6} \rho \int \mathrm{d} r\left[g(r)-g_{0}(r)\right]\left[r B_{0}^{\prime}(r)-3 \rho \partial B_{0}(r) / \partial \rho\right],
\end{aligned}
$$

which differ from the correct expressions by the additional second term in each case. It is clear that the new condition, eqs. (15), now removes this inconsistency, since $B_{0}(r)$ is constructed so that the unwanted integrals in (16) and (17) vanish. (It does not follow, of course, that the common inconsistency between virial and compressibility pressures will necessarily be lifted as well.)

To illustrate the method, the RHNC equation has been solved for the one-component plasma at $\Gamma \equiv \beta e^{2} /$ $a=20$, where $a$ is the ion-sphere radius,

$\frac{4}{3} \pi \rho a^{3}=1$,

with the hard sphere fluid as reference system. Following Rosenfeld and Ashcroft [2], $B_{0}(r)$ and $\partial B_{0}(r) / \partial \sigma$ were obtained from the Percus-Yevick equation for this model; $A_{3}^{(0)}$ in eq. (10) was computed as

$\beta A_{\xi^{(0)}} / N=\beta A^{(0)} / N-\beta A_{1}^{(0)} / N-\beta A_{2}^{(0)} / N$,

with $A^{(0)}$ the excess free energy obtained by integra- 


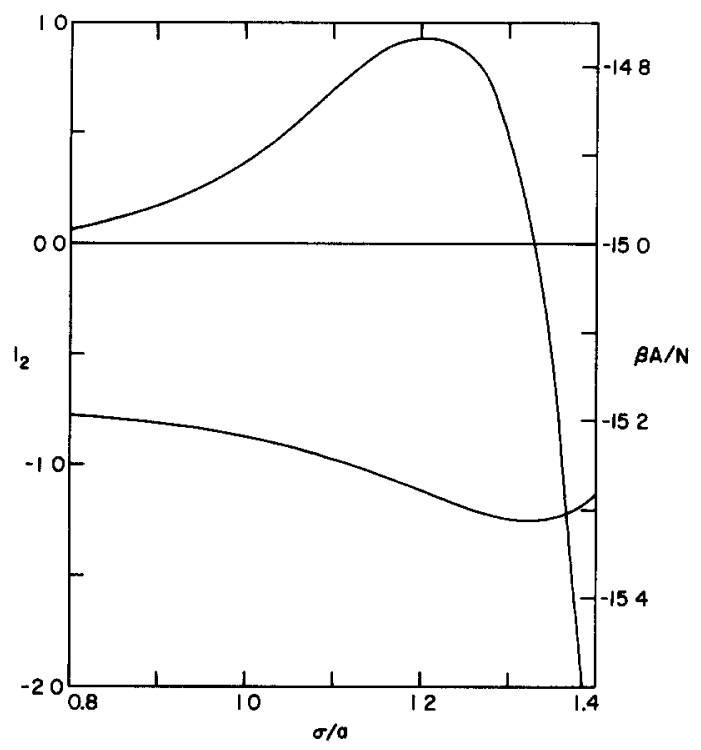

Fig. 1. Variation of the excess free energy $A$ and integral constraint $I_{2}$ as a function of hard sphere diameter for the one-component plasma at $\Gamma=20$.

tion of the Percus-Yevick virial equation of state [5], for consistency.

The results for $I_{2}$ and the free energy as functions of the hard sphere diameter $\sigma$ are shown in fig. 1. The free energy minimum is seen to be quite shallow, making direct use of this criterion sensitive to the numerical details of a calculation. The vanishing of $I_{2}$, on the other hand, is a sturdy constraint; it occurs here for $\sigma / a=1.3297$, with resulting thermodynamic values

$\beta A / N=-15.314, \quad \beta U / N=-16.711$,

$\beta \partial p / \partial \rho=-6.070$.

The corresponding numbers from a Monte Carlo calculation [6] are $-15.296,-16.667$, and -6.496 . Agreement with the first two is excellent, less so for the more sensitive inverse compressibility; pressure consistency apparently will not be achieved. [For comparison, the HNC $(\sigma=0)$ values are -14.692 , -16.538 , and -8.626 .]

More extensive results will be published elsewhere.

\section{References}

[1] F. Lado, Phys. Rev. A8 (1973) 2548.

[2] Y. Rosenfeld and N.W. Ashcroft, Phys. Rev. A20 (1979) 1208.

[3] J.S. Rowlinson, Mol. Phys. 9 (1965) 217;

F. Lado, J. Chem. Phys. 47 (1967) 4828;

P. Hutchinson and W.R. Conkie, Mol. Phys. 24 (1972) 567.

[4] J.S. Tsai, dissertation, North Carolina State University (1980), unpublished.

[5] M.S. Wertheim, Phys. Rev. Lett. 10 (1963) 321;

E. Thiele, J. Chem. Phys. 39 (1963) 474.

[6] J.P. Hansen, Phys. Rev. A 8 (1973) 3096. 